\title{
Aplicação de mapa conceitual para identificação de diagnósticos de enfermagem
}

\author{
Application of concept map for the identification of nursing diagnosis \\ Aplicación del mapa conceptual para identificación de diagnósticos de enfermería
}

\section{Greicy Kelly Gouveia Dias Bittencourt', Diego Schaurich", Maiko Marini"', Maria da Graça Oliveira Crossetti ${ }^{\mathrm{IV}}$}

' Universidade Federal do Rio Grande do Sul, Programa de Pós-Graduação em Enfermagem. (Doutoranda) Porto Alegre-RS, Brasil.

"Centro Universitário Franciscano, Curso de Enfermagem. Porto Alegre-RS, Brasil. Universidade Federal do

Rio Grande do Sul, Programa de Pós-Graduação em Enfermagem. (Doutorando) Porto Alegre-RS, Brasil.

III Prefeitura Municipal de Caxias do Sul, Secretaria Municipal de Saúde, Centro de Atenção Psicossocial Álcool e Drogas

Reviver. Caxias do Sul-RS, Brasil. Universidade Federal do Rio Grande do Sul, Programa de Pós-Graduação em Enfermagem. (Mestrando) Porto Alegre-RS, Brasil.

"v Universidade Federal do Rio Grande do Sul, Escola de Enfermagem, Departamento de Enfermagem Médico-Cirúrgica, Programa de Pós-Graduação em Enfermagem. Porto Alegre-RS, Brasil.

Submissão: 05-07-2010 Aprovação: 27-01-2012

\section{RESUMO}

Trata-se de relato de experiência cujo objetivo é o de relatar a aplicação do Mapa Conceitual como estratégia para desenvolver habilidades de pensamento crítico, na identificação dos diagnósticos de enfermagem prioritários numa situação clínica. Para tanto, apresenta-se o caso clínico fictício elaborado e o Mapa Conceitual construído com o auxílio do software Cmaps Tools. Concluiu-se que a utilização do Mapa Conceitual na identificação dos diagnósticos de enfermagem possibilitou o desenvolvimento de habilidades do pensamento crítico, devendo seu uso ser estimulado ao longo da formação acadêmica.

Descritores: Enfermagem; Diagnóstico de Enfermagem; Educação em Enfermagem.

\section{ABSTRACT}

This is an experience report, aiming at describing the application of the Concept Map as a strategy to develop critical thinking skills in the identification of priority nursing diagnoses in a clinical situation. An elaborated fictitious clinical case and the concept map, created by using the IHMC CmapTools computer program, are presented. It was concluded that the use of Concept Map to identify the nursing diagnoses led to the development of critical thinking skills; therefore, its use should be stimulated during the academic program.

Key words: Nursing; Nursing diagnosis; Education in nursing.

\section{RESUMEN}

Trata-se del relato de experiencia cuyo objetivo es relatar la aplicación del Mapa Conceptual, como estrategia para desarrollar habilidades del pensamiento crítico, en la identificación de los diagnósticos de enfermería prioritarios en una situación de clínica. Para tanto, se presenta uno caso clínico ficticio y el Mapa Conceptual construido con auxilio del software Cmaps Tools. Se concluye que la utilización del Mapa Conceptual en la identificación de los diagnósticos de enfermería posibilita el desarrollo de habilidades del pensamiento crítico, debiendo ser estimulado su uso a lo largo de la formación académica.

Palabras clave: Enfermería; Diagnóstico de enfermería; Educación en enfermería. 


\section{INTRODUÇÃO}

Ao voltar-se para a utilização do processo de enfermagem no cuidado individualizado, o enfermeiro se defronta com a prática do julgamento clínico e do raciocínio diagnóstico envolvidos na tomada de decisão sobre as ações de cuidar. Trata-se de uma prática complexa em que o profissional é responsável por assumir julgamentos, identificar diagnósticos de enfermagem, tomar decisões e ser responsável pelos resultados de suas ações. Para tanto, precisa desenvolver a habilidade de raciocínio clínico que envolve a capacidade cognitiva, o conhecimento clínico e a experiência, integrados pela percepção intuitiva da situação como um todo(1).

Sendo assim, faz-se importante enfatizar o raciocínio clínico desde a formação do enfermeiro para a identificação do diagnóstico de enfermagem, uma vez que ele extrapola a dimensão técnica do conhecimento. Nesse sentido, um aspecto importante refere-se ao ensinar e ao aprender o diagnóstico de enfermagem por se tratar de um processo interpretativo e complexo de julgamento e raciocínio clínico que envolve habilidades de pensamento crítico e de tomada de decisão ${ }^{(2-3)}$.

O pensamento crítico em enfermagem é um componente essencial da responsabilidade profissional e da qualidade da assistência. Isso porque, os pensadores críticos em enfermagem devem apresentar os seguintes hábitos mentais: confiança, perspectiva contextual, criatividade, flexibilidade, curiosidade, integridade intelectual, intuição, compreensão, perseverança e reflexão. Eles praticam as habilidades cognitivas de análise, de aplicação de padrões, de discernimento, de busca de informações, de raciocínio lógico, de predição e de transformação de conhecimento ${ }^{(4)}$.

Acredita-se que o pensamento crítico não é um método a ser ensinado, porém é entendido como um modo de pensar que pode ser apreendido e aprimorado por estratégias que desenvolvam habilidades cognitivas para pensar-se criticamente. No contexto do ensino do diagnóstico de enfermagem, entende-se que a utilização dessas estratégias auxilia no desenvolvimento de habilidades de pensamento crítico durante sua identificação. Sendo assim, diferentes estratégias podem ser utilizadas de forma associadas para desenvolvimento dessas habilidades.

$\mathrm{Na}$ literatura de enfermagem ${ }^{(5)}$, existem diferentes estratégias de ensino utilizadas para desenvolver habilidades de pensamento crítico e, dentre elas, destaca-se o Mapa Conceitual $(\mathrm{MC})$ por ser considerado uma representação gráfica que estimula a organização de conceitos e a união entre teoria e prática. Por meio do MC identificam-se conceitos-chave de um fenômeno específico que são unidos para mostrar suas conexões e fornecer informações sobre o conteúdo, estrutura e inter-relações de conhecimento no fenômeno identificado $^{(6)}$. Na prática de enfermagem, afirma-se que a utilização do MC pode estimular a visualização da interdependência conceitual e, desse modo, subsidiar o planejamento holístico do cuidado cujo objetivo é o de promover o pensamento reflexivo, a resolução de problemas e a síntese de definições ${ }^{(7)}$. Alguns autores ${ }^{(7-12)}$ argumentam a possibilidade de utilização desta estratégia no processo ensino-aprendizagem juntamente aos estudantes de enfermagem a fim de propiciar o desenvolvimento do pensamento crítico voltado para o planejamento do cuidado.

Com base numa discussão com estudantes de enfermagem sobre o pensamento crítico do enfermeiro ao identificar um diagnóstico de enfermagem, observaram-se dificuldades no desenvolvimento do raciocínio lógico para levantamento de hipóteses diagnósticas durante a resolução de um caso clínico. Nessa discussão, pontuou-se que, em algumas situações clínicas, o planejamento do cuidado se fundamenta numa percepção imediata de uma situação sem que haja um raciocínio mais minucioso para estabelecer o diagnóstico de enfermagem. Desse modo, entende-se que a percepção intuitiva do enfermeiro e sua experiência clínica são de grande relevância na interpretação e no agrupamento de dados para identificação de um diagnóstico de enfermagem. No entanto, acredita-se que o raciocínio lógico deva ser associado à intuição e à experiência clínica na tentativa de compreensão de problemas de saúde com base na percepção intuitiva e analítica do enfermeiro ${ }^{(13)}$.

Sendo assim, acredita-se que a elaboração de mapas conceituais pode auxiliar no desenvolvimento do raciocínio lógico e, consequentemente, do discernimento para a tomada de decisão quanto ao diagnóstico de enfermagem prioritário por possibilitar a visualização de conceitos inter-relacionados dispostos numa configuração significante e, assim, colaborar com o entendimento de fenômenos específicos da prática de enfermagem. Entende-se que a construção de mapas conceituais facilita o levantamento de dados, sua interpretação e seu agrupamento por meio da elucidação de proposições que dão sentido à relação dos conceitos e, desse modo, pode facilitar a identificação de diagnósticos de enfermagem.

Mediante este contexto, este artigo tem o objetivo de relatar a aplicação do mapa conceitual, como estratégia para desenvolver habilidades de pensamento crítico, na identificação dos diagnósticos de enfermagem prioritários numa situação clínica.

\section{METODOLOGIA}

Este estudo surgiu como uma proposta de trabalho final da disciplina Estratégias de Ensino do Pensamento Crítico na Enfermagem Clínica desenvolvida junto ao Programa de Pós-Graduação em Enfermagem da Escola de Enfermagem da Universidade Federal do Rio Grande do Sul, configurada como um estudo independente. Nessa disciplina, discutiu-se o arcabouço teórico-prático que subsidia o pensamento crítico na enfermagem, bem como as estratégias facilitadoras do seu desenvolvimento, tendo como mediadora da discussão a docente responsável pela mesma. Assim, foi proposta a elaboração de um caso clínico fictício a partir de um conjunto de imagens.

Para tanto, foram projetadas com o auxílio de equipamento multimídia uma série de fotos que continham imagens de partes do corpo humano acometidas por algum problema de saúde. Após este momento, foi sugerida a formação de pequenos grupos de três estudantes a fim de que fosse construído um caso clínico com base nas imagens mostradas, levando-se em consideração os aspectos bio-fisio-patológicos. Ainda, foi solicitado que os estudantes elegessem uma das estratégias de ensino apresentadas na disciplina a fim de exercitar 
a identificação dos diagnósticos de enfermagem prioritários oriundos da resolução do caso clínico.

Sendo assim, ocorreu a reunião no pequeno grupo e iniciou-se o processo de construção do caso clínico. Inicialmente, foi debatido acerca de quais imagens seriam agrupadas e utilizadas com vistas a dar sentido a história elaborada. Posteriormente, os integrantes do grupo expuseram seus pontos de vista em relação àquilo que consideravam importante estar presente no caso clínico. Concomitantemente, houve discussão e reflexão acerca de vivências e experiências prévias dos membros do grupo, que facilitaram e auxiliaram no desenvolvimento do caso clínico.

Desta forma, após a construção do caso clínico deu-se início à sua resolução com o intuito de identificar os diagnósticos de enfermagem prioritários com base na Taxonomia da North American Nursing Diagnosis Association (NANDA I) ${ }^{(14)}$ e selecionou-se como estratégia o MC para auxiliar na identificação de dados de interesse e de seus inter-relacionamentos para elaboração dos diagnósticos de enfermagem prioritários. Para tanto, recorreu-se à ferramenta CMap Tools em sua versão 5.03 que é um software desenvolvido e distribuído gratuitamente pelo Institute for Human Machine Cognition da University of west Florida, o qual permite ao usuário construir, navegar, compartilhar e criticar modelos de conhecimento representados com mapas conceituais. O CMap Tools é utilizado para fazer a autoria de mapas conceituais em que o usuário elabora e cria as figuras podendo compartilhar o conhecimento expresso com outros usuários ${ }^{(15)}$.

Os Mapas Conceituais apresentam uma organização hierárquica, porém não devem ser confundidos com organogramas ou diagramas de fluxo, pois não implicam seqüência, temporalidade, direcionalidade, nem ordens organizacionais ou de poder. Mapas conceituais são diagramas de significados, de relações significativas entre definições. Eles buscam classificar conceitos, relacioná-los e hierarquizá-los. Em princípio, o fato de dois conceitos estarem unidos por uma linha é importante porque configura que há, no entendimento de quem fez o mapa e de quem o interpreta, uma relação entre os conceitos, mas o tamanho e a forma dessa linha são, a priori, arbitrários. Não existem regras fixas para a elaboração de Mapas Conceituais, mas o importante é que exista uma compreensão do significado entre os $\operatorname{conceitos}^{(16)}$.

Assim, após a construção do mapa conceitual deste estudo, procedeu-se à elaboração dos diagnósticos de enfermagem prioritários de acordo com a NANDA I ${ }^{(14)}$ e discutiu-se acerca das habilidades de pensamento crítico necessárias na resolução do caso clínico apresentado.

\section{APRESENTAÇÃO DO CASO CLÍNICO E SUA RESOLUÇÃO}

Nesta sessão, apresenta-se um caso clínico fictício para ilustrar os sinais e sintomas visando à identificação de diagnósticos de enfermagem prioritários e mostra-se um MC para elucidar a inter-relação entre os dados identificados.

\section{Caso Clínico}

MMC, 70 anos, sexo feminino, casada, aposentada, procedente da zona rural. Ansiosa por deixar o marido de 88 anos em casa, de quem cuida. Interna sem acompanhantes, pois os filhos moram distantes. Está triste por não trabalhar na lavoura e magoada com os filhos que não a visita. Sabe que tem "pressão alta" (SIC), mas não administra medicação apesar de ter indicação para uso de anti-hipertensivo. Motivo da internação: febre recorrente. Encontra-se desidratada, com a pele ressecada e com turgor e elasticidade diminuídos. Apresenta lesão hiperemiada em exsudato na parte lateral da coxa direita e lesão hiperemiada no calcâneo esquerdo. Apresenta dificuldade de deambulação e está restrita ao leito, com dificuldades de mudar de posição. Verificados sinais vitais: $\mathrm{T}=39,3^{\circ} \mathrm{C} ; \mathrm{R}$ $=25 \mathrm{Irpm} ; \mathrm{PA}=180 \times 110 \mathrm{mg} / \mathrm{Hg} ; \mathrm{FC}=110 \mathrm{bpm}$.

A partir do caso clínico apresentado, levantaram-se dados relevantes para elaborar o MC a partir de conceitos-chave presentes no caso clínico, resultando na figura abaixo:

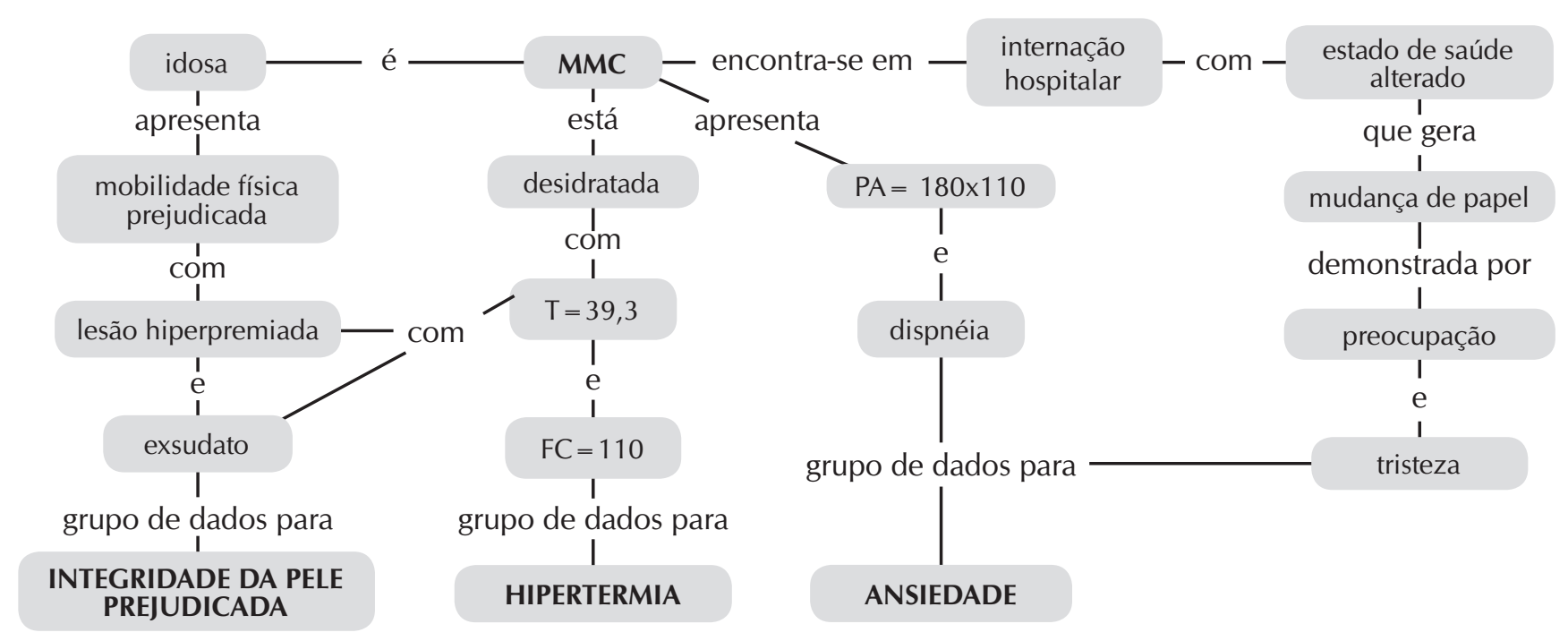

Figura 1 - Mapa conceitual com os principais dados referentes ao caso clínico 
Com base nos dados identificados, os diagnósticos de enfermagem considerados prioritários, nessa situação, foram ANSIEDADE, HIPERTERMIA e INTEGRIDADE DA PELE prejudicada como podem ser visualizados na Figura 1. A escolha pelo diagnóstico de ANSIEDADE se justifica pela mudança de função de papel que ocorreu na vida de MMC, além de mudanças no ambiente por permanecer em internação hospitalar devido ao estado de saúde alterado. Esses fatores contribuíram para que a paciente apresentasse alterações afetivas, como tristeza e preocupação, e alterações fisiológicas, como o aumento da tensão arterial e dispnéia. Assim, de acordo com a NANDA-I, tem-se o diagnóstico de ANSIEDADE relacionado à mudança de papel, evidenciada por tristeza, preocupação e aumento na tensão arterial e freqüência respiratória.

Consideraram-se a desidratação e o quadro inflamatório e infeccioso em lesão como fatores que contribuíram para HIPERTERMIA, caracterizada pelo aumento na temperatura corporal $\left(39,3^{\circ} \mathrm{C}\right)$. Com relação ao diagnóstico de enfermagem INTEGRIDADE DA PELE prejudicada, a idade da paciente MMC (70 anos) e a imobilização física contribuíram para o rompimento da superfície da pele gerando lesões.

Neste estudo, a identificação de diagnósticos de enfermagem prioritários por meio da construção de um MC possibilitou levantar sugestões de diferentes causas de um problema com vistas a reconhecer características de determinados diagnósticos. No caso apresentado, por exemplo, a paciente foi admitida num hospital com temperatura elevada. Assim, um dos conceitos centrais foi a temperatura e, em torno desse conceito, existem fatores, considerados outros conceitos, que contribuíram ou causaram a elevação da temperatura. É possível, portanto, estabelecer relações entre esses conceitos através de diagramas representativos das relações conceituais conforme se demonstrou na Figura 1.

No caso acima apresentado, foi possível identificar os conceitos-chave e estabelecer relações entre eles a fim de identificar as características apresentadas pela paciente, bem como as causas dos problemas elencados o que facilitou o levantamento de dados relevantes, sua interpretação e agrupamento para identificação de diagnósticos de enfermagem.

Por ser um instrumento organizacional metacognitivo utilizado para representar o conhecimento ${ }^{(7)}$, no ensino de enfermagem, o MC pode favorecer a organização do cuidado, uma vez que a ligação dos conceitos de forma hierarquizada e de acordo com as necessidades e prioridades de cuidar oportunizam o exame criterioso de evidências extraídas da prática clínica, assim como exige reflexão, aprendizagem e relacionamento de situações e conceitos. Além disso, fornece um conhecimento mais completo e profundo acerca das necessidades dos pacientes ${ }^{(11,12,17)}$.

Estudos demonstram a necessidade de habilidades de pensamento crítico na elaboração de um MC. Dentre elas destacam-se a capacidade de análise, de síntese e de avaliação dos dados, estimulando a criatividade para unir informações do paciente, com vistas a organizar e planejar o cuidado. Argumenta-se que essa estratégia desenvolve habilidades de pensamento crítico por consistir numa descrição gráfica do fluxo do processo de pensar que, utilizada de forma crítica e criativa, pode auxiliar no ensino de enfermagem na busca de resolução de problemas ${ }^{(9,10)}$.

Num estudo(18) relata-se que a construção de $M C$, na realização de anamnese e exame físico de enfermagem, demonstraram ser importante como sinalizador dos conceitos apreendidos pelos estudantes, além de possibilitar a integração do conhecimento novo aos anteriores e de estabelecer significados entre as definições. Outro estudo ${ }^{(19)}$, argumenta a utilidade do MC no desenvolvimento do raciocínio clínico do enfermeiro, através da resolução de casos clínicos pela sua potencialidade de demonstrar os processos cognitivos do graduando em relação à capacidade de gerar respostas eficientes na decisão/solução de problemas e a estruturação de um pensamento organizado e crítico.

No presente estudo, a construção de um MC para a resolução de um caso clínico fictício possibilitou a reflexão acerca das habilidades de pensamento crítico desenvolvidas para identificação dos diagnósticos de enfermagem prioritários. Tendo-se por base as habilidades de pensamento crítico constantes na literatura ${ }^{(4)}$, destacam-se algumas delas que foram essenciais para a elaboração dos diagnósticos de enfermagem.

Essa experiência demonstrou a necessidade de análise para levantar dados, separá-los por similaridades e diferenças e buscar dados adicionais; de raciocínio lógico para entender o agrupamento de dados relacionados entre si; de aplicação de padrões na identificação, avaliação e agrupamento de dados com base em padrões estabelecidos na literatura; bem como de conhecimento sobre a paciente e do seu contexto de vida para se compreender as necessidades subjetivas da paciente. Além disso, pontua-se a necessidade de conhecimento técnico-científico, de intuição e de experiência clínica na identificação de diagnósticos de enfermagem.

A capacidade de análise se fez presente tendo em vista a necessidade de se avaliarem as informações levantadas com o intuito de buscar evidências para a elaboração dos diagnósticos de enfermagem. Para tanto, precisou-se identificar, organizar e classificar os dados da situação em partes para considerar possíveis significados que permitiram a avaliação das informações que caracterizariam os diagnósticos de enfermagem prioritários. O raciocínio lógico contribuiu com o entendimento da relação entre os dados objetivos, subjetivos e contextuais tendo-se como base a junção de saberes teóricos e práticos para avaliação de dados relevantes que justificassem a identificação do diagnóstico de enfermagem. Foi importante, ainda, examinar a relevância dos dados e agrupá-los em padrões significativos para estabelecer a relação entre eles, julgando-os para levantar hipóteses diagnósticas e decidir acerca do diagnóstico de enfermagem adequado.

A transformação do conhecimento, oriunda da experiência clínica, representou a aplicação de conhecimentos utilizados em situações prévias para se compreender uma situação semelhante a outras já vivenciadas na prática profissional. A utilização de conhecimentos prévios demonstrou que esses saberes podem ser adaptados em outras situações, ajudando o enfermeiro a perceber diferentes modos de cuidar. 
Salienta-se a importância do conhecimento sobre o paciente com base na perspectiva contextual, uma vez que é fundamental destacar a importância de se analisar uma situação específica de determinado paciente dentro do seu contexto de vida, para que, então, haja uma valorização de suas crenças, valores e cultura. Desse modo, compreende-se que a utilização do MC para identificação de diagnósticos de enfermagem pode auxiliar no desenvolvimento de habilidades de pensamento crítico e instigar a criatividade, a curiosidade e a flexibilidade. Neste estudo, observou-se a necessidade de análise, de aplicação de padrões e de raciocínio lógico. Além disso, pontua-se que, o conhecimento técnico-científico e a intuição foram aspectos fundamentais para a compreensão de opiniões convergentes e divergentes na discussão do caso clínico apresentado.

\section{CONSIDERAÇÕES FINAIS}

A utilização do MC na identificação dos diagnósticos de enfermagem possibilitou o desenvolvimento de habilidades de pensamento crítico. A capacidade de análise e de síntese, o raciocínio lógico, a aplicação de padrões, a flexibilidade, a curiosidade e a criatividade foram algumas delas.
A identificação do diagnóstico de enfermagem requer habilidades cognitivas, atitudes, experiências e conhecimentos para que o enfermeiro faça interpretações e julgamentos acerca de dados objetivos e subjetivos do paciente. Neste contexto, o pensamento crítico se apresenta como uma habilidade essencial, capaz de orientar o enfermeiro a tomar decisões acuradas, pois a todo o momento ele interpreta as respostas humanas que demandam cuidados. Portanto, acredita-se que o MC pode ser uma estratégia que facilita o desenvolvimento de habilidades de pensamento crítico na identificação de dados e, conseqüentemente, na identificação de diagnósticos de enfermagem na prática clínica, devendo seu uso ser estimulado ao longo da formação acadêmica.

Salienta-se, neste estudo, a importância de associação de estratégias para desenvolvimento de habilidades de pensamento crítico na identificação de diagnósticos de enfermagem. A elaboração de um estudo de caso clínico fictício, a discussão em pequeno grupo e a construção do MC facilitaram a resolução do caso clínico culminando com a identificação de diagnósticos de enfermagem prioritários que, possivelmente, contribui com o planejamento da assistência de enfermagem na busca de resultados eficazes.

\section{REFERÊNCIAS}

1. Bittencourt GKGD, Crossetti MGOC. The teaching-learning process of the nursing diagnosis: literary analysis. Online Braz. J. Nurs. [serial online] 2009;8(1):1-10. available from: http://www.objnursing.uff.br/index.php/nursing/article/ view/j.1676-4285.2009.2224.

2. Corrêa CG. Raciocínio clínico: o desafio do cuidar [tese]. São Paulo (SP): Escola de Enfermagem, Universidade de São Paulo; 2003.

3. Almeida MA. Competências e o processo ensino-aprendizagem do diagnóstico de enfermagem. Rev. Bras. Enferm 2004;57(3):279-83.

4. Scheffer BK, Rubenfeld MG. A concensus statement on critical thinking in nursing. J Nurs Educ 2000;39(8):352-9.

5. Crossetti MCO, Bittencourt GKGD, Schaurich D, Tanccini T, Antunes M. Estratégias de ensino das habilidades do pensamento crítico na enfermagem. Rev. Gaúch. Enferm. 2009;30(4):732-41.

6. Taylor W. Concept mapping: a nursing model for care planning. J. Nurs. Educ. 2007;46(5):211-6.

7. Gul RB, Boman JA. Concept mapping: a strategy for teaching and evaluation in nursing education. Nurs Educ in Pratice 2006;6(4):199-206.

8. Hicks-Moore SL. Clinical concept maps in nursing education: an effective way to link theory and practice. Nurse Educ. Pract. 2005;5(6):348-52.

9. Abel WM, Freeze M. Evaluation of concept mapping in an associate degree nursing program. J. Nurs. Educ. 2006;45(9):356-64.

10. Wilgis M, Mcconnell J. Concept mapping: an educational strategy to improve graduate nurses's critical thinking skills during a hospital orientation program. J. Contin. Educ. Nurs. 2008;39(3):119-26

11. Hsu L. Developing concept maps from problem-based learning scenario discussions. J. Adv. Nurs. 2004;48(5):510-8.

12. Daley BJ, Shaw CR, Balistrieri T, Glasenapp K, Piancentine L. Concept maps: a strategy to teach and evaluate critical thinking. J. Nurs. Educ. 1999;38(1):42-7.

13. Bittencourt GKGD. Modelo teórico de pensamento crítico no processo diagnóstico em enfermagem [tese]. Porto Alegre (RS): Programa de Pós-Graduação em Enfermagem/Universidade Federal do Rio Grande do Sul; 2011.

14. NANDA International. Diagnósticos de enfermagem da NANDA: definições e classificação 2009-2011. Porto Alegre: Artmed; 2010.

15. Institute for Human and Machine. Cognition-CmapTools. University of West Florida. [cited 2010 Fev 19] available from: http://cmap.ihmc.us.

16. Moreira MA. Mapas conceituais e aprendizagem significativa. [acesso 20 Mar 2010]. Disponível em: http://www. if.ufrgs.br/ $\sim$ moreira/mapasport.pdf.

17. Staib S. Teaching and measuring critical thinking. J. Nurs. Educ. 2003;42(11):498-508.

18. Cogo ALP, Pedro ENR, Silva APSS, Specht AM. Avaliação de mapas conceituais elaborados por estudantes de enfermagem com o apoio de software. Texto Contexto Enferm. 2009;18(3):482-8.

19. De Domenico EBL, Piconez SCB, Gutiérrez MGR. Mapas conceituais para a resolução de casos clínicos como uma estratégia de ensino e aprendizagem a ser explorada na formação de enfermeiros: um ensaio. Online Braz J of Nurs 2009; 8(1):1-10. 